\title{
A comparison of anther culture derived material with single seed descent lines in Brussels sprouts (Brassica oleracea var. gemmifera)
}

J. Kubba,

B. M. Smith, ${ }^{*}$

D. J. Ockendon,*

A. P. Setter,

C. P. Werner and

M. J. Kearsey
Department of Genetics, University of Birmingham, P.O. Box 263, Birmingham B15 2TT, U.K.

* Institute of Horticultural Research, Wellesbourne, Warwick CV35 9EF, U.K.

Qualitative and quantitative studies show that diploid plants produced by anther culture (AC) from a double cross population of Brussels sprouts have arisen from spontaneously doubled haploid cells. The mean performance and distribution of lines produced by $\mathrm{AC}$ is compared to an equivalent array of inbred lines produced by single seed descent (SSD). There was no evidence of any difference in vigour between the AC and SSD samples. The tests are sufficiently sensitive to establish (with a 90 per cent confidence) that any true difference between the means, for vigour related traits, must be less than 15 per cent of the mean performance of SSD material. Additional evidence identifies genetic segregation for stability to micro-environmental factors within both the AC and SSD lines.

\section{INTRODUCTION}

In the early 1980 s predictive trials at the National Vegetable Research Station, Wellesbourne (now the Institute of Horticultural Research), indicated the possibility of producing inbred cultivars of Brussels sprouts in preference to $F_{1}$ hybrids (Smith, Rogers and Kearsey, 1985; Rogers, Kearsey and Smith, 1987). This led to the initiation of two breeding programmes to produce inbred lines; one utilizing single seed descent (SSD), and the other anther culture (AC) (Ockendon, 1984).

Of the plants regenerated from anther culture, approximately 50 per cent were haploid, 50 per cent were diploid and a few had higher ploidy levels. The diploid plants might have arisen from haploid pollen grains by spontaneous doubling of the chromosomes or from unreduced diploid cells in the anther tissue. The former developmental route provides a potentially valuable breeding tool since the products of anther culture would be fully homozygous individuals that could give rise to "instant" true-breeding lines. The alternative, however, is of no value to the plant breeder because the cultured plants would be identical clones of the anther donor.
Initial work on $\mathrm{AC}$ derived material in tobacco led to the belief that such material was less vigorous than conventionally produced inbred material (Arcia, Wernsman and Burk, 1978; Schnell, Wernsman and Burk, 1980; Brown and Wernsman, 1982). However, theoretical work by Jinks and Pooni (1981) and further comparisons of SSD and AC derived material on a variety of species such as tobacco (Jinks, Chowdhury and Pooni, 1985; Jinks, Pooni and Chowdhury, 1985) and Triticale (Charmet and Branlard, 1985) have failed to confirm this. Random samples of lines produced from a common source by SSD or by AC should have the same distributions except for the following reasons:

(1) Culture induced abnormality in the AC lines. This could be caused by somaclonal variation (Scowcroft and Larkin, 1983) or by mutational effects of the spindle inhibitor used to double the chromosome complement of the cultured haploid plantlets.

(2) Inadvertent selection amongst AC or SSD lines at any stage of production.

(3) Residual heterozygosity could remain in SSD lines owing either to insufficient generations of selfing and/or to natural selection favouring 
heterozygosity. Residual heterozygosity would inflate the within line variation of SSD material when compared to equivalent fully homozygous lines and, in the presence of positive directional dominance, would lead to a higher mean performance.

(4) Linkage disqeuilibrium. During the production of SSD lines there are opportunities for recombination during each round of inbreeding. This is not the case, however, with $\mathrm{AC}$ lines derived from $F_{1}$ pollen as the genotype is fixed after one round of recombination. This effect would produce differences in the variance of lines derived from AC and SSD and in the presence of epistatic interactions would also result in differences in the mean performance (Riggs and Snape, 1977).

The objectives of the work reported here were to determine if the diploid embryos produced from AC in Brussels sprouts developed from spontaneously doubled haploid cells and to check that the genotypic distribution of the AC material was comparable to that of a parallel set of lines produced by SSD. To achieve this, AC lines were compared with both SSD lines and nonsegregating control material for a number of production traits in field trials during 1984 and 1985. These studies constitued a small part of much larger trials. It was possible in 1984 to collect data on an individual plant basis and therefore to compare variance components. In 1985 only whole plot records were possible and these were used to compare the mean performance of the two groups.

Starch gel electrophoresis was also used to provide a qualitative test of genotypic uniformity.

\section{MATERIALS AND METHODS}

The 1984 trial contained 20 random SSD lines which had been selfed for three generations and $41 \mathrm{AC}$ lines. Both sets of lines were derived from the double cross population produced by crossing two $F_{1}$ hybrids, Pinnacle and Nym. Anthers were cultured from four donor plants taken from the Pinnacle $\times$ Nym double cross using the methods described by Ockendon (1984). Haploids, spontaneous diploids and plants with higher ploidy levels were produced. Each of the AC lines included in this study were produced by self pollinating a spontaneous diploid plant. Other than restricting the sample of lines to spontaneous diploids no selection of the AC material was made. In the field each line was represented by a singlerow plot of 12 plants. The trial also contained 12 plots of control parental material consisting of two plots of the Pinnacle $\times$ Nym double cross, one plot of Pinnacle $F_{1}$, one of Nym $F_{1}$, one of each of the four inbred parents and 4 additional plots of $\mathrm{GA}_{1}$ (one of the parental lines). This trial formed part of a continuing breeding programme for which the SSD lines were planted in one block while the AC lines and the controls were grown in an adjacent block; this design structure precludes the comparison of the mean performance of the two groups.

The 1985 trial contained 24 SSD lines and 43 AC lines randomised in a single block with each line represented by a single plot of 12 plants. This material comprised part of a larger experiment to assess the performance of SSD material (Werner, Setter, Smith, Kubba and Kearsey, in press). Different random samples of lines were tested in 1984 and 1985.

In both years seed was sown in $125 \mathrm{~cm}^{3}$ peat blocks in mid-March. The plants were transplanted to the field in mid-May and harvested between October and January. When mature the central ten plants from each plot were harvested on the same day, the sprout buttons were stripped from the stems mechanically and the buttons graded by hand into marketable and waste fractions.

In 1984 the following two characters were scored in the field on two consecutive days in September on an individual plant basis:

(1) Largest leaf length $(\mathrm{mm})$.

(2) Largest leaf width $(\mathrm{mm})$.

and a further five characters were assessed on the day the plot was harvested; again on an individual plant basis:

(3) Waste weight $(\mathrm{g})$.

(4) Marketable weight ( $\mathrm{g}$ ).

(5) Stem length $(\mathrm{mm})$.

(6) Sprout number.

(7) Sprout quality (subjectively scored on a scale of $1-5,5$ being high quality).

For the 1985 data the leaf characters were not assessed and all other characters were recorded only on a whole plot basis. One further character is presented:

(8) Total weight $(\mathrm{g})$.

In order to compare the means and particularly the variances of the SSD and AC material the data should be approximately normally distributed with no correlation between the mean and variance. Epistasis or $\mathrm{G} \times \mathrm{E}$ interactions could cause such non-normality. In order to decide which scale transformation to use the following criteria for rescaling the 1984 data were applied to the ten control plots of parental material: 
(i) The chosen scale minimised the correlation between means and within-plot variances. The scales tested included logarithm transformations and a range of power transformations as shown by Rogers et al. (1987).

(ii) A range of transformations were also checked, using Levene's test (Levene, 1960), for homogeneity of the within-plot variance over all the control genotypes.

In addition to these, variance ratio tests were carried out to check that the within-plot variances of the segregating double cross were greater than for the $F_{1} s$ and parental inbred lines.

Comparable scale transformations of the 1985 data were not possible because this trial was recorded only on a whole plot basis.

An electrophoretic analysis was carried out on leaf samples taken from five plants from each of $24 \mathrm{AC}$ lines using the techniques of Arus and Orton (1983). Plants and lines were chosen at random from the material growing in the field during 1984. Approximately 25 samples were run on each starch gel, including the two $F_{1}$ parental lines as controls. The gels were stained for phosphoglucoisomerase (PGI) activity and a three-allele system (a,b, c) was established, with Pinnacle carrying the "b" and " $c$ " alleles and Nym carrying the " $a$ " and "c" alleles. No SSD material was examined using electrophoresis.

\section{RESULTS}

Electrophoretic analysis revealed that all 5 plants in each of the $24 \mathrm{AC}$ lines scored were homozygous at the PGI locus. Furthermore all five plants from each line were of the same genotype, there being a total of four lines with genotype "aa" and 20 lines with genotype "cc". For each line, therefore, the probability that the parental plant (from anther culture) was heterozygous for this locus is $\left(\frac{1}{2}\right)^{5}=$ $3 \cdot 1$ per cent. The absence of "bb" types is probably caused by a sampling effect incurred because the AC lines were derived from only four plants taken from the double cross population. Two of these sources only give rise to "cc" $\mathrm{AC}$ lines and may therefore have been homozygous ("cc") for the PGI locus. These two sources account for nine of the $\mathrm{AC}$ lines assessed as "cc". The two remaining anther donors gave rise to both "aa" and "cc" AC lines and, therefore, must have been "ac" heterozygotes for the PGI locus.

Tests of the scale were carried out for the 1984 data using the 10 control plots of non-segregating material. The means and variances for four of the characters were significantly correlated $(P<5$ per cent) on the measured scale. These charactersleaf length, waste weight, marketable weight and sprout number-were transformed by taking the square root of the measured data. For each of these characters this was close to the optimum power transformation and in all cases resulted in nonsignificant correlations between the means and variances.

For the square root transformations of leaf length, waste weight and sprout number the Levene's analysis identified significant heterogeneity $(P<5$ per cent) for the variation measured within the various non-segregating control families. No scale transformation could be found which removed the mean-variance correlation and this heterogeneity simultaneously. For every character except leaf length, the double cross variances were greater, as expected, than those of the non-segregating generations on both the untransformed and square root scales. The variance for leaf length within the $F_{1}$ plots was greater than that for the double cross on the untransformed scale whilst on the square root scale the expected ranking was restored. The square root transformations were therefore retained.

Heterogeneity of the variation observed within different genotypes may be attributed to the effects of genotype by environment interactions $(\mathrm{G} \times \mathrm{E})$ acting at the level of the micro-environment (within-plot). Therefore, it was assumed that the genotypes assessed (including all SSD and AC lines) might have different environmental variances and that this should be taken into account when testing for differences in variance between the SSD and AC samples. Therefore a Levene's hierarchical analysis of variance (Levene, 1960) was conducted to take account of any heterogeneity within the samples.

The results of the Levene's hierarchical analysis of variance are given in table 1 . The analysis uses absolute deviations of the individual scores from the line mean. The between lines item represents a test for heterogeneity of the micro-environmental error within the AC and SSD samples. There was no significant difference between the $\mathrm{AC}$ and SSD samples for this item for any of the characters and therefore in table 1 the pooled mean square is presented. This item was significant for all characters, indicating the presence of genotype by microenvironmental interactions. For the characters waste weight, marketable weight, stem length, sprout number and sprout quality there was no significant difference between the SSD and AC samples. Therefore for these characters there was 
Table 1 Levene's hierarchical analysis of variance of the 1984 data. The within line deviations of the SSD and AC samples are compared using variance ratio tests. Probability levels given are $*<5$ per cent, ${ }^{*} * 1$ per cent

\begin{tabular}{|c|c|c|c|c|c|c|c|c|c|c|c|c|c|c|c|}
\hline \multirow[b]{2}{*}{ Item } & \multirow[b]{2}{*}{$\mathrm{df}$} & \multicolumn{2}{|c|}{$\begin{array}{l}\text { Leaf } \\
\text { length }\end{array}$} & \multicolumn{2}{|l|}{$\begin{array}{l}\text { Leaf } \\
\text { width }\end{array}$} & \multicolumn{2}{|c|}{$\begin{array}{l}\text { Waste } \\
\text { weight }\end{array}$} & \multicolumn{2}{|c|}{$\begin{array}{l}\text { Market. } \\
\text { weight }\end{array}$} & \multicolumn{2}{|c|}{$\begin{array}{l}\text { Stem } \\
\text { length }\end{array}$} & \multicolumn{2}{|c|}{$\begin{array}{l}\text { Sprout } \\
\text { number }\end{array}$} & \multicolumn{2}{|c|}{$\begin{array}{l}\text { Sprout } \\
\text { quality }\end{array}$} \\
\hline & & M.S. & $P$ & M.S. & $P$ & M.S. & $P$ & M.S. & $P$ & M.S. & $P$ & M.S. & $P$ & M.S. & $P$ \\
\hline Between samples & 1 & $1 \cdot 38$ & $*$ & 1111 & $*$ & $32 \cdot 13$ & ns & $13 \cdot 70$ & ns & 607 & ns & 0.39 & ns & 0.024 & ns \\
\hline Between lines & 59 & $0 \cdot 32$ & $*$ & 278 & $* *$ & $9 \cdot 40$ & $* *$ & $14 \cdot 44$ & $* *$ & 2057 & $* *$ & $1 \cdot 68$ & $* *$ & 0.119 & $*$ \\
\hline Residual & 713 & 0.23 & & 172 & & $4 \cdot 00$ & & $4 \cdot 83$ & & 920 & & $0 \cdot 26$ & & 0.088 & \\
\hline
\end{tabular}

no evidence of any systematic difference of the micro-environmental (within-plot) variation between these groups.

In the analysis of leaf length and leaf width the between samples item was just significant with probabilities of 4.9 and 4.3 per cent respectively. The SSD lines possessed the larger deviations. A closer inspection of these two characters showed that in both cases the deviations of the AC lines were not significantly different from the controls but that the SSD lines had significantly larger (3.0 and $3 \cdot 1$ per cent) mean deviations when compared with the control genotypes. Table 2 shows that for every character the mean deviation was larger in the SSD sample than in the AC sample. These observations confirm that the $\mathrm{AC}$ lines show less variation between individuals than the SSD lines and indicate the presence of residual heterozygosity within the incompletely inbred SSD material.

Table 3 provides a comparison of the variation between lines for the AC and SSD samples for both 1984 and 1985. In both years each line was represented by a single plot of ten recorded plants but the 1984 data are based upon individual plant records and rescaled data whereas the 1985 data are based upon whole plot records retaining the raw scale. For all characters and in both years the between lines item was significant at the 1 per cent level when tested against the available error term. In 1984 no significant differences in the between line mean squares could be detected between the SSD and AC samples. This pattern was repeated in 1985 for each character with the exception of waste weight which showed a highly significant

Table 2 Mean absolute deviations for the SSD and AC samples

\begin{tabular}{llllllll}
\hline Sample & $\begin{array}{l}\text { Leaf } \\
\text { length }\end{array}$ & $\begin{array}{l}\text { Leaf } \\
\text { width }\end{array}$ & $\begin{array}{l}\text { Waste } \\
\text { weight }\end{array}$ & $\begin{array}{l}\text { Market } \\
\text { weight }\end{array}$ & $\begin{array}{l}\text { Stem } \\
\text { length }\end{array}$ & $\begin{array}{l}\text { Sprout } \\
\text { number }\end{array}$ & $\begin{array}{l}\text { Sprout } \\
\text { quality }\end{array}$ \\
\cline { 5 - 8 } SSD & 0.711 & 18.1 & 2.86 & 2.62 & 37.1 & 0.587 & 0.427 \\
AC & 0.608 & 15.2 & 2.37 & 2.30 & 34.9 & 0.533 & 0.413 \\
s.e.d. & 0.0498 & 1.46 & 0.269 & 0.333 & 3.98 & 0.1136 & 0.0302 \\
$(\mathrm{df}=59)$ & & & & & & & \\
\hline
\end{tabular}

Table 3 Comparison of SSD and AC between line mean squares in 1984 and 1985. 1984 data is based upon individual records and 1985 data upon whole plots. The significance given is for a two-tailed variance ratio test. Probability levels as in table 1

\begin{tabular}{|c|c|c|c|c|c|c|}
\hline & \multicolumn{3}{|l|}{1984} & \multicolumn{3}{|l|}{1985} \\
\hline & SSD & $\mathrm{AC}$ & $P$ & SSD & $\mathrm{AC}$ & $P$ \\
\hline Leaf length & $16 \cdot 88$ & $27 \cdot 47$ & ns & - & - & - \\
\hline Leaf width & 5666 & 6060 & ns & - & - & - \\
\hline Waste weight & $197 \cdot 0$ & $168 \cdot 3$ & $\mathrm{~ns}$ & 2,661 & 12,274 & $* *$ \\
\hline Market weight & $178 \cdot 7$ & $179 \cdot 0$ & ns & 14,919 & 22,425 & ns \\
\hline Total weight & - & - & - & 16,312 & 15,419 & ns \\
\hline Stem length & 1225 & 1527 & $\mathrm{~ns}$ & 118.4 & $151 \cdot 5$ & ns \\
\hline Sprout number & $10 \cdot 70$ & $9 \cdot 51$ & ns & $126 \cdot 0$ & $175 \cdot 0$ & ns \\
\hline Sprout quality & $2 \cdot 65$ & $3 \cdot 19$ & ns & 0.333 & 0.323 & ns \\
\hline (df) & (19) & $(40)$ & & (23) & $(42)$ & \\
\hline
\end{tabular}


Table 4 Comparison of the mean performance of SSD and AC lines in 1985. The difference and the standard error of the difference (SED) is given together with the significance level based upon Students $t$ tests. Probability levels as in table 1

\begin{tabular}{|c|c|c|c|c|c|}
\hline & SSD & $\mathrm{AC}$ & SSD-AC & SED & $P$ \\
\hline Waste weight (g) & $85 \cdot 0$ & $132 \cdot 9$ & $-47 \cdot 9$ & $19 \cdot 91$ & $*$ \\
\hline Market weight $(\mathrm{g})$ & $318 \cdot 3$ & $266 \cdot 3$ & $52 \cdot 0$ & $33 \cdot 81$ & ns \\
\hline Total weight $(\mathrm{g})$ & $403 \cdot 4$ & $399 \cdot 2$ & $4 \cdot 2$ & $32 \cdot 22$ & ns \\
\hline Stem length ( $\mathrm{mm})$ & $68 \cdot 53$ & $65 \cdot 85$ & $2 \cdot 68$ & 2.908 & ns \\
\hline Sprout number & $39 \cdot 71$ & $39 \cdot 10$ & 0.61 & $3 \cdot 053$ & ns \\
\hline \multirow[t]{2}{*}{ Sprout quality } & $2 \cdot 48$ & $2 \cdot 51$ & -0.03 & 0.146 & ns \\
\hline & & & & $(65 \mathrm{df})$ & \\
\hline
\end{tabular}

difference with the AC sample having the larger variation between lines.

The character means, shown in table 4, were derived from the data collected in 1985. Student's $t$ tests showed no significant differences between the means of the AC and SSD lines for any of the characters apart from waste weight in which the AC lines had a larger mean $(P=1.9$ per cent $)$. Closer inspection will show that the mean total yields of the SSD and AC samples were very close and that it is with respect to the partitioning of the yield into waste weight and marketable weight that the discrepancy has arisen. This pattern follows that observed for the analysis of the between line variation from 1985 where, although total yield showed equality between the SSD and AC groups, waste weight, and to a lesser extent marketable weight, behaved quite differently. It would therefore appear that some factor may be involved which, whilst not disturbing the relative vigour of these groups, plays a role in the partitioning of the yield into waste and marketable fractions.

Table 4 suggests that, for the characters of greatest agronomic importance, the differences between the mean performances of $\mathrm{AC}$ and SSD lines were small. But the identification of a nonsignificant difference may only reflect the use of a very insensitive test. In order to give an indication of the power of the comparisons the distribution of the observed difference between AC and SSD means was used to determine a 90 per cent confidence interval, symmetric about zero. The length of this interval, expressed as a percentage of the mean SSD performance, is presented in table 5 . It can be seen that for the characters of general vigour, total weight, stem length and sprout number, the data support the conclusion (with a 90 per cent confidence) that the true difference between the AC and SSD material is no greater than 15 per cent of the mean SSD performance and may, of course, be considerably less. Only for waste weight
Table 5 Maximum true difference between SSD and AC means expressed as a percentage of the mean SSD performance. Based upon the 1985 data with a 90 per cent confidence

Max. difference (per cent SSD)

\begin{tabular}{lr}
\hline Waste weight & $77 \cdot 1$ \\
Market weight & 29.9 \\
Total weight & 14.7 \\
Stem length & $9 \cdot 6$ \\
Sprout number & 13.8 \\
Sprout quality & 9.6 \\
\hline
\end{tabular}

and to a lesser extent marketable weight is there a possibility that the difference could be greater than this.

In addition to the mean and variance of the full distribution plant breeders are concerned with the breeding potential of lines in the upper tail. For total yield five of the AC lines ( 11.6 per cent) and three of the SSD lines ( 12.5 per cent) raised in 1985 were superior to $\mathrm{GA}_{1}$ (an established inbred line which was included as a reference genotype in the 1985 triall). For average quality one AC line ( $2 \cdot 4$ per cent $)$ and two SSD lines $(8 \cdot 3$ per cent) outperformed $\mathrm{GA}_{1}$. Chi-squared tests showed that neither of these comparisons nor similar ones for waste weight, marketable weight, stem length or sprout number were significant at the 5 per cent level.

\section{DISCUSSION}

The electrophoresis investigations showed that for all of the lines examined every individual was homozygous. The hierarchical Levene's analysis showed for every character that the mean absolute deviation within lines was less for the AC sample 
than for the comparable group of SSD lines. These two separate tests provided very strong evidence that the AC lines studied were derived from embryos which developed following the spontaneous doubling of haploid cells.

The Levene's hierarchical analysis also showed that for each character in both the SSD and AC samples there was a considerable degree of heterogeneity between families for the within line variance. This represents the effects of interactions between micro-environmental factors and genotypes. In breeding terms this means that in this cross there was considerable genetic segregation for micro-environmental stability and therefore the practice, in conventional breeding, of selecting for uniformity will not only tend to select the more homozygous lines but also those with an inherently more stable genotype.

Having ascertained that the $\mathrm{AC}$ material was homozygous it was possible to consider the relative performance of the AC and SSD material. With the exception of waste weight there was no evidence of any difference between the $A C$ and SSD samples neither with respect to the mean performance not to the variation between lines. The significant differences that were detected for waste weight would appear to be qualitative differences, affecting the partitioning of total yield into waste and marketable fractions rather than a difference in vigour. These data therefore provide good evidence for the absence of any serious differences in vigour between inbred lines of Brussels sprouts obtained by SSD and those produced by the spontaneous doubling of antherderived haploids. There is a strong possibility that the larger variation between $\mathrm{AC}$ lines which was observed during 1985 for waste weight was a secondary effect of the difference between the means for this character. Setter (1988) showed in a full treatment of the 1985 trial that the means and between line variances of seven sets of pure lines raised alongside the material presented in this paper were strongly correlated for the waste weight character $(r=0.918, P=0.004)$. The possibility cannot be ruled out that, for waste weight, there was a true difference between the $\mathrm{AC}$ and $\mathrm{SSD}$ samples caused by the presence of inadvertent selection, the differential segregation of interacting linked genes in the coupling phase (Jinks, Chowdhury and Pooni, 1985) or by sampling variation. Sampling could affect the comparison because the anther culture lines were derived from four double-cross individuals whereas each SSD line was produced from a different individual taken at random from the double-cross population. However, it is also possible that the difference in the mean represents a chance event (with six characters under study the probability of at least one of these showing a significant difference at the 5 per cent level is 27 per cent) and that the large difference in the between line variation reflects the effects of a meanvariance correlation. With respect to this latter point it should be noted that no discrepancy occurred in 1984 and that in 1985, owing to the use of whole plot scores, it was not possible to carry out a scale transformation.

The studies presented demonstrate that in the Brussels sprouts crop the spontaneous diploid products of anther culture do perform comparably to an equivalent sample of inbred lines produced by conventional methods, both with respect to the mean and distribution of the lines, for all the major characters.

Acknowledgements We thank Mr K. Lavery for his valuable technical assistance during the course of this work. Computing facilities were made available for this work at both the Computer Centre, University of Birmingham and at the IHR. J.K. and C.P.W. were in receipt of an AFRC Link award and A.P.S. a SERC studentship.

\section{REFERENCES}

ARCiA, M. A., Wernsman, E. A. AND bURK, L. G. 1978. Performance of anther-derived dihaploids and their conventionally inbred parents as lines, in $F_{1}$ hybrids and in $F_{2}$ generations. Crop Sci, 18, 413-418.

ARUS, P. AND ORTON, T. J. 1983. Inheritance and linkage relationships of isoenzymes in Brassica oleracea. $J$. Hered. 74, 405-412.

BROWN, J. S. AND WERNSMAN, E. A. 1982. Nature of reduced productivity of anther-derived dihaploid lines of flue-cured tobacco. Crop Sci., 22, 1-5.

CHARMET, G. AND BRANLARD, G. 1985. A comparison of androgenetic doubled-haploid and single seed descent lines in Triticale. Theor. Appl. Genet., 71, 193-200.

JINKS, J. L., CHOWDHURY, M. K. U. AND POONI, H. S. 1985 Comparison of the inbred lines derived from a hybrid of tobacco (burley $x$ fiue cured) by dihaploidy and single seed descent. Heredity, 55, 127-133.

JINKS, J. L. AND POONI, H.S. 1981. Properties of pure-breeding lines produced by dihaploidy, single seed descent and pedigree breeding. Heredity, 46, 391-395.

JINKS, J. L., POONI, H. S. AND CHOWDHURY, M. K. U. 1985. Detection of linkage and pleiotropy between characters of Nicotiana tabacum using inbred lines produced by dihaploidy and single seed descent. Heredity, 55, 327-333.

LEVENE, H. 1960. Robust test for equality of variances. In Olkin, I., Churye, S. G., Hoeffding, W., Madow, W. G. and Mann, H. G. (eds) Contributions to Probability and Statistics, Stanford University Press, Stanford, pp. 278-292.

OCKENDON, D. J. 1984. Anther culture in Brussels sprouts (Brassica oleracea var. gemmifera). I. Embryo yields and plant regeneration. Ann. Appl. Biol., 105, 285-291. 
RIGGS, T. J. AND SNAPE, J. W. 1977. Effects of linkage and interaction in a comparison of theoretical populations derived by single seed descent and doubled haploid methods. Theor. Appl. Genet., 49, 111-115.

ROGERS, W. J., KEARSEY, M. J. AND SMITH, B. M. 1987. The feasibility of producing inbred rather tha $F_{1}$ hybrid cultivars in Brussels sprouts: Predictions from early generations. Ann. Appl. Biol., 111, 677-688.

SCHNELL, R. J., WENSMAN, E. A. AND BURK, L. G. 1980. Efficiency of single seed descent vs. anther-derived dihaploid breeding methods in tobacco. Crop Sci., 20, 619-622.

SCOWCROFT, W. R. AND LARKIN, P. J. 1983. Somaclonal variation and genetic improvement of crop plants. In Nugent, J. and O'Connor, M. (eds) Better Crops for Food, Ciba Foundation, London, pp. 173-197.
SETter, A. P. 1988. Predicting the Results of Breeding Programmes in Outbreeding Species. Ph.D. Thesis, Faculty of Science, University of Birmingham.

SMITH, B. M., ROGERS, W. J. AND KEARSEY, M. J. 1985. The feasibility of producing inbred rather than $F_{1}$ hybrid cultivars in Brussels sprouts: a preliminary genetical analysis and choice of material for inbreeding. Ann. Appl. Biol., 107, 87-99.

WERNER, C. P., SETTER, A. P., SMITH, B. M., KUBBA JENAN AND KEARSEY, M. J. Performance of recombinant inbred lines in Brussels sprouts (Brassica oleracea var. gemmifera). Theor. Appl. Genet. (In press). 